ISSN: 2521-0874 (Print)

ISSN: 2521-0505 (Online)

CODEN: AIMCCO

REVIEW ARTICLE

\title{
A COMPARATIVE STUDY OF CROWD COUNTING AND PROFILING THROUGH VISUAL AND NON-VISUAL SENSORS
}

\author{
Jugal Kishor Gupta ${ }^{1}$, S.K. Gupta ${ }^{2}$ \\ ${ }^{1}$ Research Scholar, IFTM University, Moradabad, India. \\ ${ }^{2}$ Associate Professor Department of Computer Science \& Engineering BIET, Jhansi, India. \\ *Corresponding Author email: jugal.kishor@vidya.edu.in, guptask biet@rediffmail.com
}

This is an open access article distributed under the Creative Commons Attribution License, which permits unrestricted use, distribution, and reproduction in any medium, provided the original work is properly cited

\section{ARTICLE DETAILS}

\section{Article History:}

Received 15 November 2018 Accepted 17 December 2018 Available Online 07 January 2019

\section{ABSTRACT}

In this paper we present a comparative critical study of visual and non-visual sensors used in crowd behavior analysis. The understanding of crowd has main impact of the analysis how much they support the system is the key factor of the analysis. The surveillance and security prospects are the main feature for crowd that can make system easy and gives better result. The visual sensors that have been widely used are wireless sensor network, computer vision, smart camera, sensor fusion and few more; and the non-visual sensors are regarding the call, IEEE 802,11 signals measurement, Smart Evactrack, Social Network and Bluetooth etc. This comparative study identified the different analysis of crowd behavior and after analysis we show which technique is better to another one. The smart devices are used now days for surveillance and gives better result in crowd behavior analysis.

\section{KEYWORDS}

Video Surveillance, Crowd Density, Crowd Analysis, Counting by detection, population profiling.

\section{INTRODUCTION}

A large group of peoples are called crowd who are available in a particular area. In general place like airport, daily market, bus stations, railway station like places is very necessary and difficult task to identify the unwanted person over thousands of peoples. It is very difficult for human to count the head and identified over thousands of gathering manually. Such things motivate us as researcher to develop a automated system for identifying and capturing any type of suspicious behavior of a person in such places where more than thousands peoples are available.

As now a day's such type of analysis are going on and researchers are worked on safety and security issues that cover the surveillance on crowd. Target facing, Suspense detection, recognized behavior, emotions recognition and crowd density estimation etc. these are possibility for automation of the system [1-9].

Now a day's whole research is going towards automation and sufficient progress are notify. And we find in this analysis a lot of work are pending in visual crowd analysis in particular detection of clear picture of suspected behavior [10]. We saw a general framework for the same where we saw the different mode of suspected are traced and identified and analyzed in all ways so that the clear picture founds.

By the large usability of smart electronics devices and different non-visual sensors, so that non-visual sensing-based research persons have been done their research on crowd analysis [11].

To better knowing the crowd dynamic, non-visual sensing has best ones in terms of advantage in compare with others like density estimation and localization. Smart devices facilitate on-human body sensing which is not geographically restricted to a particular structured area. Today's smart devices are already equipped with sensors like accelerometers, motion sensors. We have a lot of sensors which allows us to differentiate the behavior of a particular person in a crowd [10].

In this paper we mainly focus on the technique which are used the visual and non-visual sensors with particular focus on detection and estimation of a suspected behavior person identification. This paper organized as follows. In section 2 defines the visual and non-visual sensors in section 3 available technique in crowd analysis. In section 4 privacy issues discussed in section 5 the issues are discussed related to facing challenges in different techniques. In section 6 the dataset is discussing which are used in such techniques in section 7 comparing all techniques and in section 8 we present the conclusion.

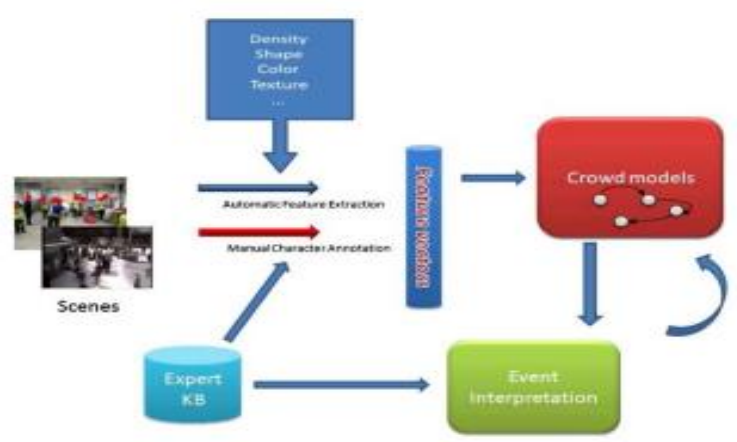

Figure 1: Shows the general framework for crowd behavior tracking system. 
This figure shows the general model for crowd estimation. In this model the sensor senses the unwanted behavior of the crowd and the features of collected data has been analyzed and send it to the features vectors that can be refined and send to the crowd model which also have the input density shape color featured and expert system. That can define the data by using expert interpretation which is managed manually initially. Due to the smart sensor which is used in smart devices it gives exact values to the feature's vectors

\section{SMART SENSING DEVICE VISUAL AND NON-VISUAL}

Now days the usability of smart devices dramatically increases that can increase the tracking of any person very easily and people are very much keen to share information on social media or any social platform [6]. Smart devices like mobile phone having GPS sensor, health monitoring system having IoT based sensor, smart watches having WiFi devices, light sensor, magnetic sensor, motion sensor, IR sensor which are generally used by smart devices that are easily tractable.

The sensor which are used in mobile devices are having different parameter depends on their type and they collectively sensing the data and sends upon their needs. Smart crowd sensing refer to the huge variety of sensing models where user sensing the computing devices and collect the data and contribute to require data for different useful application like object tracking, counting of person in crowd, behavior analysis of object and anomaly detection.

Smart sensing devices play a very good role for crowd behavior analysis. The researcher those are used the signal processing system have achieved a good successes but now a day days sensors play a very good role in all aspects. they play a good role in whether forecasting visualizing of crowd etc. but today researcher have more focus on sensor which gives accurate value and frequently. This things motivate us to be used such sensors and find out the crowd behavior in very easy way and result will be more predictive.

\section{TECHNIQUE IN CROWD ANALYSIS}

\subsection{Tracking and Behavioral detection}

This is main and important features of the crowd analysis that can help in the process and make easy. Firstly, the useable sensor senses the data and that type of data should be analyzed for future use. The tracking is not so easy because of moving object in such type of crowd. In visual system having abs tackle like raining, ice, object shadows, side effect and having so much voice of crowd. More work already done, and some model gives very good result in this field. Some motion model having a good result in this field. Few authors' works on dense crowd some work on individual some work on single image and some work on multiple cameras [12].

As a non-visual sensor-based research having very strong improvement on the field of crowd analysis. Some researcher used data fusing technique where they find very good results [7]. Research where camera are used they gives result but having some time consuming to identifying individuals because of moving objects. But we see that the mobile based devices having capability to identified object in real time and produce an accurate result in time and their desirable values.

\subsection{Head Count and density estimation of the crowd}

The head count and measurement of density are combined used by researcher to easily identification. In any panic situation is very important to identified head count in crowd so that the unwanted person identified. Wide varieties of sensors used for counting of heads like vision sensor, US sensor, IR Sensor motion sensor etc, for different angles mostly cameras are used. In some research we saw that the cameras are used for surveillance as well as head count uses. This technique should work like stadium, metro stations, bus stations etc.

In the report presented by yogamina having the error rates only $2.05 \%$ used the concept of video sequencing and detection flows [12]. A very different approach was applied to counting the peoples those are waiting the bus at bus stops according to that they can assign the ticket to the person in a particular buses and also shows that waiting time period that also can solve the problem of face counting in crowd.

On the other hand non, visual sensors the researcher followed different approaches for head count. They used RFID, BT sensor and WiFi for the purpose of counting and analysis of density. Sndip has develop a system for counting which are totally based upon the WiFi system [12]. In this system people moving in between access point they are counted as head. A very different system developed by SVT those are used to count the head by mobile phones with accuracy of $98 \%$, and system work in a very efficient way and give better result with no error rate [9].

Considering the above all method we reach the conclusion that mobile system is better option in all available option for counting and density estimation. Video based system having some improvement for giving better result.

\section{ISSUES IN PRIVACY}

Issue of privacy in crowd bases researchers very crucial in all aspect especially in mobile counting or social media-based counting. In crowd some users are not agreed to share their personal information in the system. But it can be possible if we asked the crowd to share their information by smart phone they will be agree as we show our interest in surveillance only to avoid the unwanted things. We saw the people are less interested to share the location but in public place for the purpose of their beneficial they will allow for the same. So that for their safety they allow and share their location in crowd by using smart device sensors.

\section{FACING CHALLENGES IN DIFFERENT TECHNIQUES}

In smart system-based counting, peoples are usually avoided in all aspect they are not agreed to share the personal location in the system so that is main challenge for head counting. Most of the system are used the data which are given by peoples in crowd which are give not guarantee to share so that we give the some inceptive or some offers to the crowd so that they can share the information which are very necessary to head counting.

A Wi-Fi based where they allow crowd to play a geographical game and based on that they collect the information from the users. They allow only playing in wifi enabled area so that crowd may be bound and also it will be a challenge for that.

The sound and information is collected in very specific regions. So that it is best way to provide the incentive those peoples those are involved and participate and share location and data. The participant receives reward if they participate only. So, our conclusion is if we want a smart system based counting then we must gives some incentive to the users in crowd.

\section{USED TOOLS AND DATASETS}

Choices of dataset are very important part of any research or we say that the key of crowd behavior research is datasets. Some datasets are usually available in the internet that can be used by researcher like UCSD, PET and CAVIAR. For smart devices-based dataset it also a challenge for researcher but also some system is available to provide dataset for mobile sensing devices. Vision based tools are available for sensing the devices.

\section{COMPARISONS OF ALL TECHNIQUE WITH RESULT ANALYSIS}

For different approaches are used for crowd analysis by using visual and non-visual sensors. Some time crowd sensing structured and some time unstructured and depends upon sensors. We saw that visual or non-visual sensor used anywhere but they depend upon the scene of crowd. We also cannot be ignoring the challenges which are faced by all researchers. All most every researcher said clearly in his research that they face directly or in directly challenge in their state of art. The comparative study we will shown by a given below table.

\begin{tabular}{|l|l|l|l|l|}
\hline Types of Sensors & Scope & Approach & Structured & Unstructured \\
\hline
\end{tabular}




\begin{tabular}{|c|c|c|c|c|}
\hline \multirow[t]{2}{*}{$\begin{array}{l}\text { Non-Visual (Crowd } \\
\text { Detection) }\end{array}$} & MIX & $\begin{array}{l}\text { Optical Approach, Dominant } \\
\text { Motion }\end{array}$ & Yes & NO \\
\hline & Single & $\begin{array}{l}\text { Social Force Model, Function of } \\
\text { Energy }\end{array}$ & Yes & NO \\
\hline \multirow[b]{2}{*}{$\begin{array}{l}\text { Visual (Crowd } \\
\text { Counting/TA) }\end{array}$} & Based on Network & Based on bandwidth & Yes & NO \\
\hline & Individual Device & Use of cluster & Yes & Yes \\
\hline \multirow{2}{*}{$\begin{array}{l}\text { Non-Visual (Crowd } \\
\text { Detection) }\end{array}$} & MIX & Advance ML and Optical flow & Yes & NO \\
\hline & Single & Detection Flowing & Yes & NO \\
\hline \multirow{2}{*}{$\begin{array}{l}\text { Visual (Crowd } \\
\text { Counting/DA) }\end{array}$} & Based on Network & Recording of data & Yes & Yes \\
\hline & Individual Device & Social sites/Bluetooth & Yes & Yes \\
\hline
\end{tabular}

\section{CONCLUSION}

In this paper we present the all possible research comparison and how best we perform in this area that gives the motivation towards crowdbased research. Theoretical comparative study done in this paper that clearly mentions the tracking and detection techniques that can be choose in your research it may be helpful. Smart phones and social networkingbased system are sufficient to deduct ant tracked the unwanted behavior in the crowd.

\section{REFERENCES}

[1] Aware person detection and tracking in crowds in 2011 International Conference on Computer Vision, Nov 2011, pp. 2423-2430.

[2] Roggen, D., Wirz, M., Troster, G., Helbing, D. 2011. Recognition of crowd behavior from mobile sensors with pattern analysis and graph clustering methods. Networks and Heterogeneous Media, 6(3), 521-544.

[3] Nanhore, S.D., Bartere, M.M. 2013. Mobile phone sensing system for health monitoring. International Journal of Science and Research (IJ SR), 2 (4), 252-255.

[4] Guo, B., Wang, Z., Yu, Z., Wang, Y., Yen, N.Y., Huang, R., Zhou, X. 2015. Mobile crowd sensing and computing: The review of an emerging humanpowered sensing paradigm. New York, NY, USA, 48, 7:1-7:31.

[5] Lane, N.D., Miluzzo, E., Lu, H., Peebles, D., Choudhury, T., Campbell, A.T. 2010. A survey of mobile phone sensing. IEEE Communications magazine, 48 (9), 140-150.
[6] Ravindranath, L.S., Thiagarajan, A., Balakrishnan, H., Maddenl, S. 2012. Code in the air: Simplifying sensing and coordination tasks on smartphones. Proceedings of the Twelfth Workshop on Mobile Computing Systems \& Applications, New York, NY, USA, Hot Mobile 12, 4:1-4:6.

[7] Ganti, R.K., Ye, F., Lei, H. 2011. Mobile crowd sensing: current state and future challenges. IEEE Communications Magazine, 49 (11), 32-39.

[8] Yilmaz, A., Javed, O., Shah, M. 2006. Object tracking: A survey. New York, NY, USA, Dec. 38.

[9] Yang, T., Zhang, Y., Tong, X., Zhang, X., Yu, R. 2013. A new hybrid synthetic aperture imaging model for tracking and seeing people through occlusion. IEEE Transactions on Circuits and Systems for Video Technology, 23 (9), 1461-1475.

[10] Ge, W., Collins, R.T., Ruback, R.B. 2012. Vision-based analysis of small groups in pedestrian crowds. IEEE transactions on pattern analysis and machine intelligence, 34 (5), 1003-1016.

[11] Wirz, M., Kelly, E.M., Franke, T., Camilleri, V., Montebello, M., Roggen, Lukowicz, P., Troster, G. 2013. Using mobile technology and a participatory sensing approach for crowd monitoring and management during large-scale mass gatherings. Co-evolution of Intelligent Sociotechnical Systems, 61-77. Springer.

[12] Kjaergaard, M.B., Wirz, M., Roggen, D., Troster, G. 2012. Detecting pedestrian flocks by fusion of multi-modal sensors in mobile phones. Proceedings of the 2012 ACM Conference on Ubiquitous Computing, New York, NY, USA, 2012, Ubi Comp 12, pp. 240-249. 